\title{
Investigation of Formal Methods Based on Algebraic Specifications for Sensor Systems
}

\author{
Masaki Nakamura, Akira Urashima, Tomoji Toriyama, Takahiro Seino, and Kokichi Futatsugi
}

\begin{abstract}
We propose a way to use CafeOBJ algebraic specification language for describing formal specifications of sensor systems and for verifying desired properties to be satisfied by the systems. We give a way to not only verify specifications but also generate test cases from the results of verifications. Although we take a simple mobile device application using an acceleration sensor as an example, our approach may be applied to practical sensor systems.
\end{abstract}

Index Terms-Algebraic specifications, formal methods, sensor systems, test case generation.

\section{INTRODUCTION}

Sensor systems are used in variety areas including medical and healthcare systems. For example, we are developing a sensor system for detecting "Pointing and Calling" by nurses in hospitals, with a small wireless acceleration and gyro sensor and a wireless microphone attached to the arm and the head of a nurse respectively [1]. We are also developing other medical and healthcare systems: a visualization and analysis system for chair-rise action of hemiplegia patients, an evaluation system for car driving of encephalopathy patients. Because of a wide use of sensor systems including life-critical or safety critical systems in recent years, it is needed to establish a way to develop reliable sensor systems with formal methods. In this article, we study a way to develop reliable sensor systems with a formal specification language called CafeOBJ.

CafeOBJ is a most advanced formal specification language with many advanced features for describing and verifying equational specifications, for example, flexible mix-fix syntax, powerful and clear typing system with ordered sorts, parametric modules and views for instantiating the parameters, module expressions, and equational logic by rewriting used as a powerful interactive theorem proving system for verification with proof scores [2]. Test case generation from proof scores of CafeOBJ specifications has been studied in [3]. Our benefits of this study are to formalize sensor system especially for our case studies of medical and healthcare systems in algebraic specifications and to apply the test case generation to the sensor systems.

Manuscript received August 15, 2013; revised October 15, 2013. This work was supported in part by Grant-in-Aid for Scientific Research (S) 23220002 from Japan Society for the Promotion of Science (JSPS)

Masaki Nakamura, Akira Urashima, and Tomoji Toriyama are with the Department of Information Systems Engineering, Toyama Prefectural University, Japan (e-mail: masaki-n@pu-toyama.ac.jp).

Takahiro Seino is with Faculty of International Social Studies, Maebashi Kyoai Gakuen College, Japan.

Kokichi Futatsugi is with the School of Information Science, Japan Advanced Institute of Science and Technology (JAIST).
Fig. 1 shows an outline of how to create a program code together with test codes (in Objective-C) from CafeOBJ specification and verification results, called proof scores.

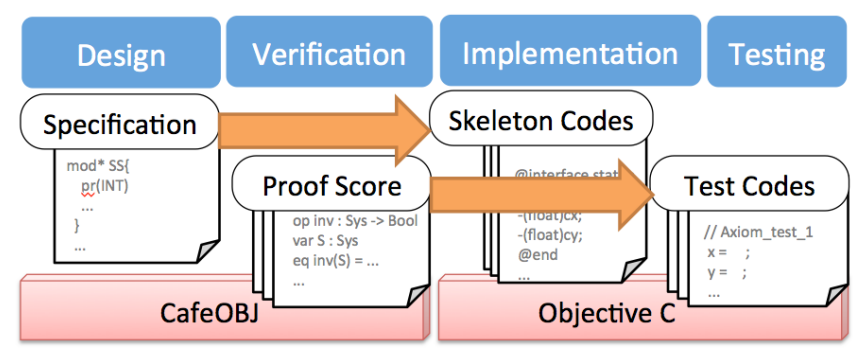

Fig. 1. Development process.

\section{An EXAmple OF Sensor System}

We take an example of small applications using an acceleration sensor, which has one square box and a ball image, and change the position of the ball according to a tilt, which can be detected by acceleration sensor, of the device, i.e. when the device is tilted, the ball moves to that direction, which looks like a rolling ball on a board (Fig. 2). We assume a coordinate $(x, y)$ means the position of the ball where $(0,0)$ stands for the leftmost-topmost of the screen, $(200,0),(0$, $300)$ and $(200,300)$ stand for the rightmost-topmost, the leftmost-bottommost and the rightmost-bottommost of the screen respectively.

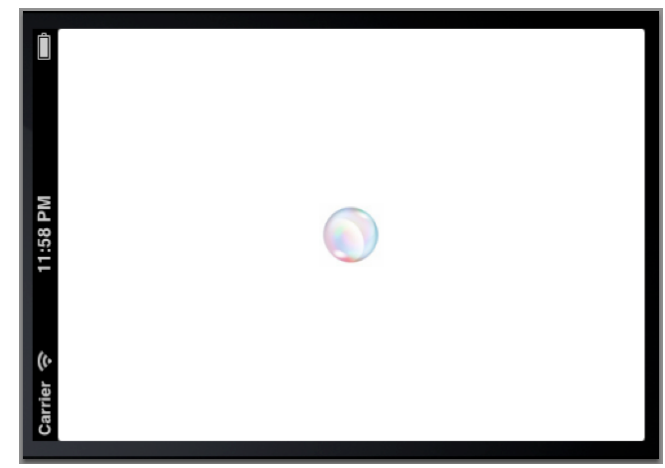

Fig. 2. Screenshot of the rolling ball application.

A typical implementation is to update the position of the ball for some interval time, e.g. $20 \mathrm{~ms}$. Then, it takes the values of the acceleration sensor every $20 \mathrm{~ms}$, and updates the position of the ball. The ball is not allowed to go outside of the screen. We use Objective-C and Xcode IDE for developing iOS applications.

\section{OTS/CAFEOBJ SPECIFICATION FOR SENSOR SYSTEMS}

We give a CafeOBJ specification for the above example in the model of the observational transition system (OTS), 
which has several case studies of formal description and verification of practical systems, for example, secure protocols [4], e-government system [5] and so on.

CafeOBJ specifications consist of modules. Our OTS/CafeOBJ specification consists of a single module named SS as follows:

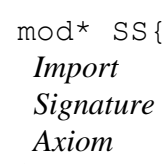

where Import, Signature and Axiom are the import part, the signature part and the axiom part of the module respectively.

In the import part, other modules, predefined or built-in, can be declared as imported modules of the module. In SS, we import a built-in module INT with the protect mode, denoted by pr (INT). INT specifies integers with ordinary operator. Since SS, includes pr(INT), we can use integers: ..., $-2,-1,0,1,2, \ldots$, and integer expressions, for example, $1+2,3>2$, and so on, in the signature and the axiom parts in SS.

In the signature part, sorts and operators are declared. A sort is a name of entities of the same type. In OTS models, a system's state is specified via observations and transitions without any explicit structures, like, sets, lists, and so on. In OTS/CafeOBJ specification, a sort of system's states is declared between "* [" and "] *", and called a hidden sort. In SS, we declare a hidden sort Sys denoting states of the sensor system. An operator is defined on sorts. An operator has its arity and sort, where an arity is a string of sorts. In CafeOBJ, an operator $f$ is declared like op $f$ : arity $->$ sort. ops is used for declaration of plural operators in one line. bop and bops are used for declaration of behavioral operators in OTS models. A behavioral operator is a special operator whose arity includes the hidden sort. When the sort of a behavioral operator is not hidden, it is called an observation. When it is hidden, it is called a transition. In OTS models, a state is identified by the observed values with the observations and a transition is defined by the change of the observed values from the pre-state to the post-state of the transition. The following is the signature part of SS:

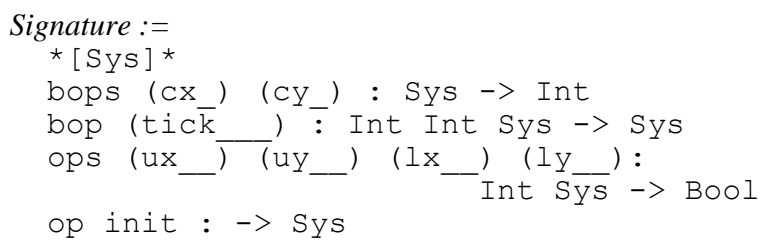

where $\mathrm{Cx}$ and $\mathrm{Cy}$ are observations for the coordinate $(x, y)$ of the ball. The term (or expression) " $\mathrm{CX} \quad \mathrm{S}$ " means the value $x$ of the coordinate $(x, y)$ at the state S. tick is a transition, and the term "tick X Y S" means the state after an interval time with the sensor's acceleration values $X$ and $Y$ of $S$. The operators $u x, u y, I_{x}$ and $l_{y}$ are auxiliary operators defining the meaning of tick, which give conditions for checking borders of the screen. The operator init is the initial state of the system.

In the axiom part, equations to be satisfied are described. An equation is declared with eq. An equation with a condition is declared with ceq. The following equations are definition of the initial state:

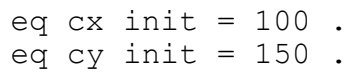

where the terms "cx init" and "cy init" are the values observed by observations $\mathrm{cx}$ and $\mathrm{cy}$ at the initial state init. The above equations mean that $x$ - and $y$-coordinates of the initial state are 100 and 150 respectively. The transition tick is defined via observations. The following is one of the equations for tick:

$$
\begin{aligned}
\text { ceq } \mathrm{cX} \text { (tick X Y S) } & =(\mathrm{cx} S)+\mathrm{X} \\
& \text { if } \mathrm{ux} X \mathrm{~S} \text { and lx X S }
\end{aligned}
$$

where $\mathrm{X}, \mathrm{Y}$ and $\mathrm{S}$ are variables, and the equation means that the $x$-coordinate increases by $\mathrm{X}$ when the sensor's acceleration value for $x$-coordinate is $\mathrm{X}$ if the range conditions for the $x$-coordinate hold. Note that the term "tick X Y S" stands for the result state of applying tick with the acceleration values $X$ and $Y$. The conditions $u x$ and $1 x$ are defined by the following equations:

$$
\begin{aligned}
& \text { eq ux } x S=(\text { cx } S)+x<=200 \text {. } \\
& \text { eq Ix X } S=(\text { cx } S)+x>=0 \text {. }
\end{aligned}
$$

where the first equation means that the condition "ux X S" is the statement that the value of the ball's $x$-coordinate is less than or equal to 200 after adding $\mathrm{X}$ or not. The second one is it is greater than or equal to zero. Thus, the above conditional equation holds if the value of the $x$-coordinate is between zero and 200 after adding $X$ or not.

In order for the ball not to go outside of the screen, it is needed to give the meaning of tick for the case that ux (or lx) does not hold as follows:

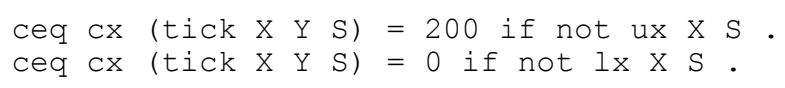

where the first (resp. the second) equation means that if the $x$-coordinate of the ball goes beyond 200 (resp. below zero), then it is forced to be set just 200 (resp. zero).

The observed values for the $y$-coordinate can be given similarly as follows:

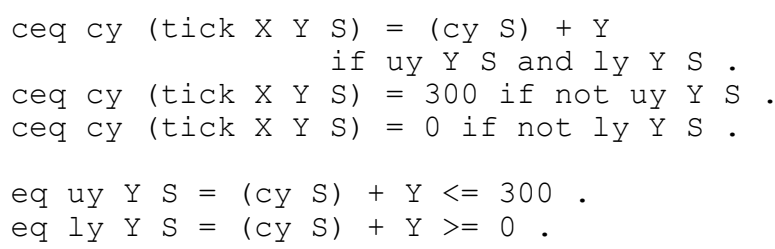

\section{SPECIFICATION EXECUTION}

CafeOBJ supports specification execution based on the term rewriting theory. A given term is reduced by applying equations in the axiom part. In reduction, each equation is regarded as a left-to-right rewrite rule. For a given term, an instance of the left-hand side of an equation is replaced with the corresponding right-hand side when the corresponding condition is reduced into true; the replacement is called a rewriting. Reduction is done by rewriting repeatedly until it cannot. The following is an example of reduction by 
CafeOBJ languages (some parts are omitted):

CafeoBJ> red in SS : cx (tick -10 20 init). (90) : NzNat

where the input term "cx (tick -10 20 init)" means the $x$-coordinate of the ball after applying tick with -10 and 20 for $x$ - and $y$-coordinates respectively to the initial state. It should be $(90,170)$ from the initial coordinate $(100,150)$, and the above output is 90 as expected. The input and output of reduction is equivalent in all model of the specification. Thus, the above reduction can be regarded as a proof of the equation $\mathrm{Cx}$ (tick -1020 init) $=90$.

\section{VERIFICATION}

By equational reasoning as above, more complicated proofs can be described and verified semi-automatically. In this example, we try to verify the following invariant property: for an arbitrary state reachable from the initial state by applying tick, the coordinate of the ball is inside of the screen. The property can be given formally as follows:

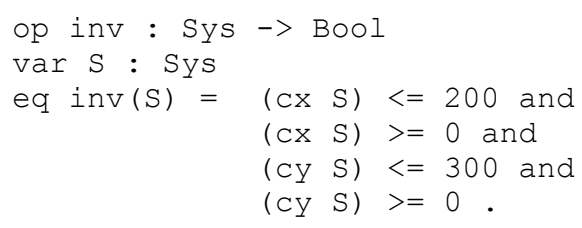

where our goal is to prove inv $(s)$ for all reachable state $s$.

The notion of proof scores is useful to prove invariant properties [2]. A proof score consists of several proof passages. A proof passage consists of reduction with (if needed) premises given by equations with constants. A proof of invariant property is given by the induction on the structure of reachable states. The base step is a proof for the initial state:

$$
\text { red inv(init) . }
$$

The initial coordinate is $(100,150)$ and it satisfies the predicate inv. CafeOBJ returns true for this reduction. The induction hypothesis is given as follows:

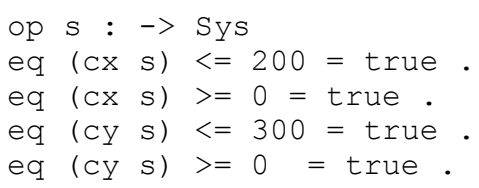

where the operator (constant) s stands for an arbitrary state satisfying the invariant predicate. Note that the conjunction of the above four equations is equivalent to $\operatorname{inv}(\mathrm{s})$. Since there is only one transition tick in SS, the next state from $\mathrm{s}$ can be represented by "tick x y s". Under the assumption of the I.H., the induction step is done by checking the following reduction:

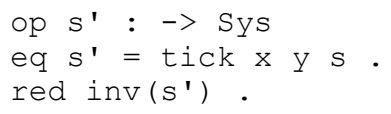

If this reduction of inv $\left(S^{\prime}\right)$ returns true, which means that $\operatorname{inv}\left(s^{\prime}\right)$ implies $\operatorname{inv}\left(s^{\prime}\right)$ is proved, then the proof by the induction was completed since every reachable state from init in SS can be represented as "tick $x_{n} y_{n}$ (tick $x_{n-1}$ $y_{n-1} \ldots\left(\operatorname{tick} x_{0} y_{0}\right.$ init)...) " for some $x_{0}, y_{0}, x_{1}, y_{1}, \ldots$ in Int.

Unfortunately, the above reduction does not return true since CafeOBJ supports only simple equational reasoning Thus, we need to give appropriate case splitting for this induction proof.

For example, when the following equations are assumed, the reduction returns true:

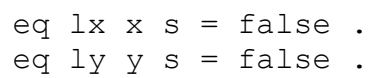

Let LX and $\sim \mathrm{LX}$ be the formulas "Ix $\mathrm{x} s=$ true" and "Ix x s = false". Let LY, LY, UX, UX, UY and UY be the formulas defined in the same way. The above equations correspond to $\sim \mathrm{LX}$ and $\sim \mathrm{LY}$. The meaning of the reduction returning true under the assumption is a proof of the formula: $\sim \mathrm{LX}, \sim \mathrm{LY}$, inv (s) implies inv(s'). To complete the induction proof, we need to prove all the other cases, e.g. $\sim \mathrm{UX}, \sim \mathrm{UY}$, inv ( $\mathrm{s}$ ) implies $\operatorname{inv}\left(\mathrm{s}^{\prime}\right)$ ). We have the completed proof score that consists of ten proof passages as follow:

1) $\operatorname{inv}($ init)

2) LX, UX, LY, UY, inv (s) implies inv ( $\mathrm{s}^{\prime}$ )

3) $\sim$ LX, LY, UY, inv (s) implies inv ( $\left.s^{\prime}\right)$

4) $\sim \mathrm{UX}, \mathrm{LY}, \mathrm{UY}$, inv (s) implies inv ( $\left.\mathrm{s}^{\prime}\right)$

5) LX, UX, LY, inv (s) implies $\operatorname{inv}\left(\mathrm{s}^{\prime}\right)$

6) $\sim \mathrm{LX}, \sim \mathrm{LY}, \operatorname{inv}(\mathrm{s})$ implies inv ( $\left.\mathrm{s}^{\prime}\right)$

7) $\sim \mathrm{UX}, \sim \mathrm{LY}, \operatorname{inv}(\mathrm{s})$ implies inv $\left(\mathrm{s}^{\prime}\right)$

8) LX, UX, $\sim \mathrm{UY}$, inv (s) implies inv ( $\left.\mathrm{s}^{\prime}\right)$

9) $\sim \mathrm{LX}, \sim \mathrm{UY}, \operatorname{inv}(\mathrm{s})$ implies $\operatorname{inv}\left(\mathrm{s}^{\prime}\right)$

10) $\sim \mathrm{UX}, \sim \mathrm{UY}$, inv (s) implies inv $\left(\mathrm{s}^{\prime}\right)$

where all proof passages return true. Note that they cover all cases, i.e. the conjunction of the proof passages from 2 to 10 is equivalent to the formula "inv (s) implies $\operatorname{inv}\left(\mathrm{s}^{\prime}\right)$ ".

\section{IMPLEMENTATION}

In [3], a way to generate a skeleton code from an OTS/CafeOBJ specification has been given. We extend it for sensor systems. From the observations, the interface named state is defined as follows:

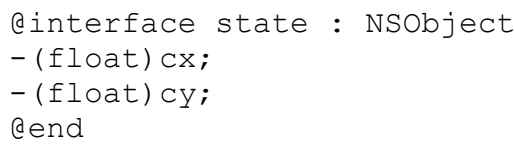

where state has a method corresponding to each observation. Then, the main interface is defined as follows:

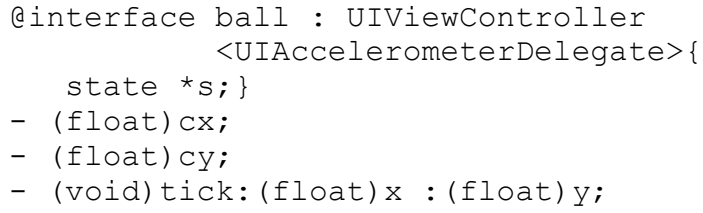


dend

where the class, named ball, has the instance variable $s$ of state, and the methods $c x$ and $c y$ for the observations and tick for the transition of the input specification. The methods for the observations just return the corresponding value of state, e.g., (float) $\mathrm{cx}\{$ return $[\mathrm{s} \mathrm{Cx}]$; $\}$. The method tick is called in a method called every $20 \mathrm{~ms}$ as follows:

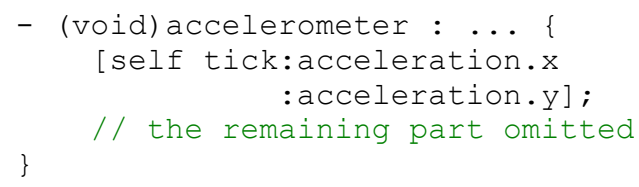

where accelerometer is a method called every $20 \mathrm{~ms}$ in the sensor system (we omit the details).

An implementer is expected to fill in the definition part of the methods for transitions (tick in this example) together with other implementation if needed, e.g., instance variables, their getters and setters for state and so on. For example, tick may be implemented as follows:

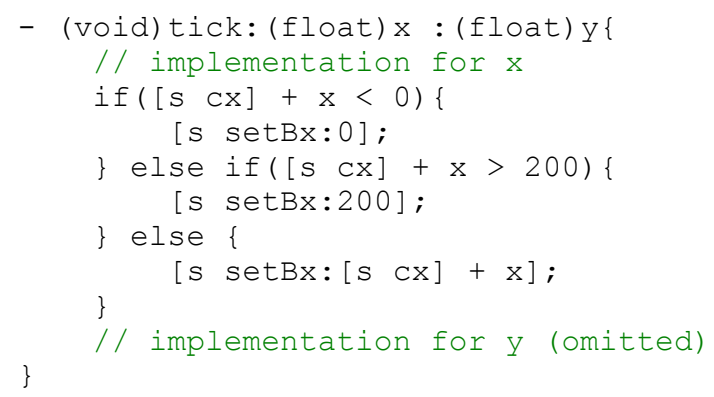

Note that the above implementation of tick is not generated from the specification, and is expected to be tested such that it satisfies the invariant property.

\section{TESTING}

Formal verification for an invariant property guarantees that it has been mathematically proved that the specification satisfies the invariant property. However, there is a gap between a specification and its implementation, for example, the former assumes INT to be the (infinite) set of all integers, though the latter may use 32-bit integers. Thus, even if the specification is verified formally, we still need to check whether its implementation satisfies the properties. For this purpose, we give a way to generate test cases for the property. The literature [3] gives not only a way to generate a skeleton code but also test cases generated from the specification and its proof scores.

To obtain a test case, we give a translation from operators except behavioral ones used in the input specification to methods in the implementation. For SS, the addition operator + , the comparison operators $>=$ and $<=$ of INT, the auxiliary operators $u x, u y, l_{x}$ and $l y$ are translated into the following methods respectively:

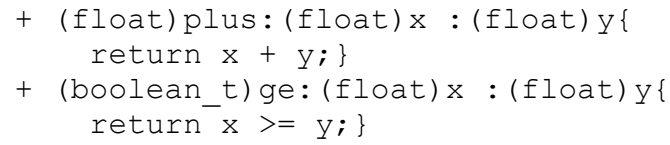

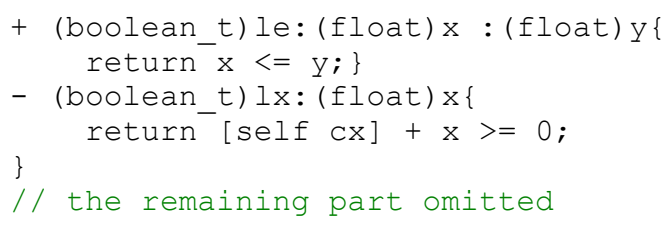

Then, we have a way to translate the equations in the specification, and the invariant property and an assumption in each proof passage into the test codes.

\section{A. Axiom Tests}

Testing for axioms is obtained from the specification's equations defining the meaning of transitions. In $S S$, the transition tick is defined by six equations. From each of these equations, we have a skeleton code of test cases that test the equation under the condition. Consider the following equation:

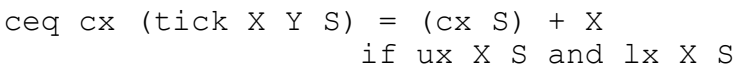

The following skeleton code of test cases (we use SenTestingKit package built in Xcode IDE) is generated from the above equation:

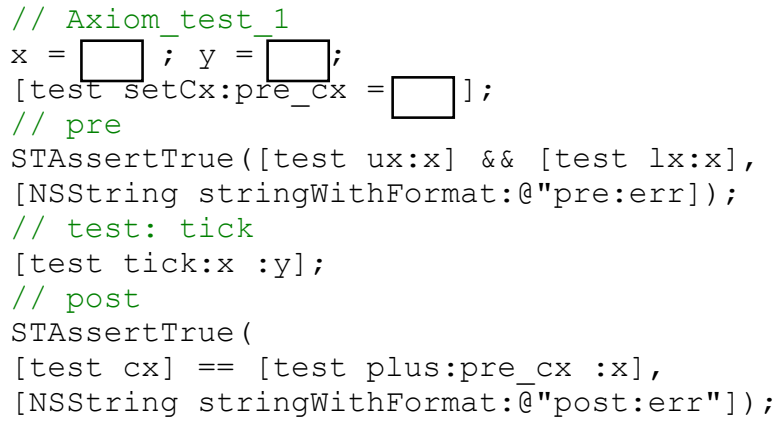

The test code consists of four parts. The first part is the preparation part, where a tester fills an appropriate value for each variable in the boxes. The second one is an assertion for the pre-condition, where the variables are tested such that they satisfy the condition generated from the condition of the equation. The third one is the test execution of the transition. The last one is an assertion for the post-condition, where the current value of the $x$-coordinate is tested such that it is equivalent with the sum of the $x$-coordinate of the pre state and the value of the variable $x$, which comes from the right-hand side of the equation.

\section{B. Invariant Tests}

As we showed in the previous section, a proof score for an invariant property consists of several proof passages. Each proof passage for the induction step corresponds to an assumption (case splitting). The assumptions are considered to be important for verifying the invariant property, and they are expected to be useful for tests of the invariant property in an implementation. The following is the output from the proof passage 2 for the case of LX, UX, LY, UY in the previous section:

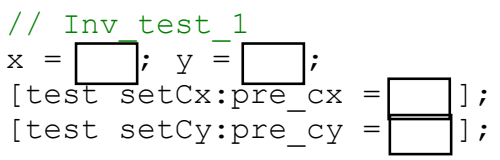


/ / pre

STAssertTrue ( [test $1 x: x] \& \&$ [test ux:x] \&\& [test

$1 \mathrm{y}: \mathrm{y}] \& \& \quad[$ test uy:y],

[NSString stringWithFormat: ( "pre:err"]);

STAssertTrue ([test inv],

[NSString stringWithFormat: ("pre:err2"]);

/ / test: tick

[test tick:x :y];

/ / post

STAssertTrue ([test inv],

[NSString stringWithFormat: ("post:err"]);

In the pre-condition part, two assertions exist. The one assertion checks the assumption of the proof passage; LX, UX, LY, UY, and the other one checks the invariant property for the variables filled by a tester. After executing the transition tick, the invariant property is checked again.

\section{CONCLUSION}

We applied formal methods with CafeOBJ language to development of a simple acceleration sensor system. We gave a way to model the sensor system in OTS models, and to describe a CafeOBJ specification. Some invariant property was verified by using the notion of proof scores. Lastly, we gave a way to generate test cases that may be appropriate for testing the invariant property in the implementation.

In the previous section, we only showed a way to generate test cases for logic unit tests with a tester explicitly setting variables. To give a way to obtain test cases for application tests by using real sensor values is one of our future work.

The example we provided in this article is so simple, though we can extend our approaches for more practical system. To apply our proposed methods to practical sensor system is another one of our future work. One of the candidates is the medical and healthcare systems we have developed, e.g. in [1].

\section{REFERENCES}

[1] A. Urashima, M. Nakamura, T. Toriyama, J. Oshima, M. Nakagawa and T. Nomura, "Preliminary Results of Pointing and Calling Detection System for Nurses," in Proc. the 11th Asia Pacific Conference on Computer Human Interaction (APCHI 2013).

[2] K. Futatsugi, D. Gaina, and K. Ogata, "Principles of proof scores in CafeOBJ," Theoretical Computer Science, vol. 464, pp. 90-112, 2012.

[3] M. Nakamura and T. Seino, "Generating Test Cases for Invariant Properties from Proof Scores in the OTS/CafeOBJ Method," IEICE Transactions, vol. 92-D, no. 5, pp. 1012-1021, 2009.

[4] I. Ouranos, K. Ogata, and P. S. Stefaneas, "Formal analysis of TESLA protocol in the timed OTS/CafeOBJ method," in T. Margaria and B. Steffen, ed., ISoLA of Lecture Notes in Computer Science, vol. 7610, no. 2, pp.126-142, 2012.

[5] W. Q. Kong, K. Ogata, and K. Futatsugi. "Towards reliable e-government systems with the OTS/CafeOBJ method," IEICE Transactions, vol. 93-D, no. 5, pp. 974-984, 2010.

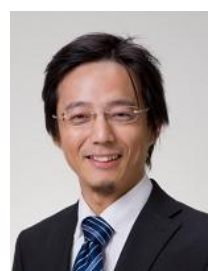

Masaki Nakamura was born in Japan in 1974. He is an associate professor at Department of Information Systems Engineering, Faculty of Engineering, Toyama Prefectural University from 2011. He received his $\mathrm{PhD}$ in information science from Graduate School of Information Science, Japan Advanced Institute of Science and Technology (JAIST) in 2002. He was an assistant professor at Graduate School of Information Science, JAIST from 2002 to 2008, and an assistant professor at School of Electrical and Computer Engineering, College of Science and Engineering, Kanazawa University from 2008 to 2011. His research interest includes software engineering, formal methods, algebraic specification and ubiquitous systems.

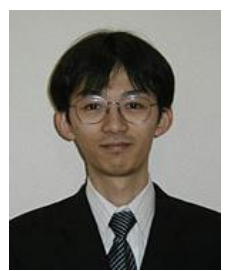

Akira Urashima was born in Japan in 1970. He is an associate professor at Department of Information Systems Engineering, Faculty of Engineering, Toyama Prefectural University from 2011 . He received his $\mathrm{PhD}$ in engineering from Graduate School of Engineering, Kyoto University in 1999. He was an assistant professor at Toyama Prefectural University from 1999 to 2011. His research interests focus on human activities identification with ubiquitous sensors and its application.

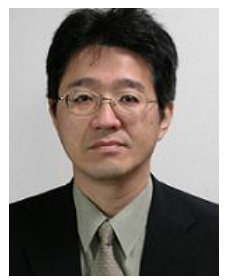

Tomoji Toriyama was born in Japan in 1961. He received B.E. and M.E. degrees from Toyama University and a Ph.D. from Toyama Prefectural University in Japan. In 1987, he joined Nippon Telegraph and Telephone Corporation (NTT). From 2007-2008, he was a group leader of the Knowledge Science Laboratories at ATR. He is currently a professor at Department of Information Systems Engineering, Faculty of Engineering, Toyama Prefectural University from 2008. His research interests include Large-Scale Integration (LSI) circuit architecture design methodology, human interfaces and human interactions. $\mathrm{He}$ is a member of the Human Interface Society and Institute of Electronics Information and Communication Engineers (IEICE).

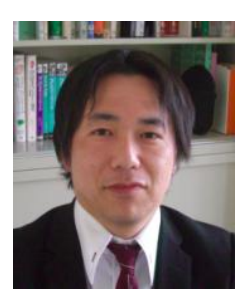

Takahiro Seino was born in Japan in 1975. He is an assistant professor at School of International Social Studies, Maebashi Kyoai Gakuen College. He received his $\mathrm{PhD}$ in information science from Graduate School of Information Science, Japan Advanced Institute of Science and Technology (JAIST) in 2003. His research interests include combining formal methods and ontology for large-scale information systems. He is occupied on some actual projects which built up large-scale information systems applying his research results.

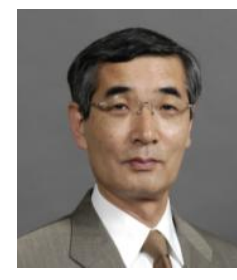

Kokichi Futatsugi was born in Japan in 1948. He is a professor at Graduate School of Information Science, JAIST (Japan Advanced Institute of Science and Technology). Before getting a full professorship at JAIST in 1993, he was working for ETL (Electrotechnical Lab.) of Japanese Government and was assigned to be Chief Senior Researcher of ETL in 1992. His research interests include formal methods, system verifications, software requirements/specifications, language design, concurrent and cooperative computing. His primary research goal is to design and develop new languages which can open up new application areas, and/or improve the current software technology. His current approach for this goal is CafeOBJ formal specification language. CafeOBJ is multi-paradigm formal specification language which is a modern successor of the most noted algebraic specification language OBJ. 